\title{
Active surveillance characterizes human intratumoral T cell exhaustion
}

\author{
Ran You, ${ }^{1,2}$ Jordan Artichoker, ${ }^{2,3}$ Adam Fries, ${ }^{2,3}$ Austin W. Edwards, ${ }^{2,3}$ Alexis J. Combes, ${ }^{2}$ Gabriella C. Reeder, ${ }^{2}$ Bushra Samad, ${ }^{2}$ \\ and Matthew F. Krummel $\left.\right|^{1,2,3}$
}

'Department of Pathology, ${ }^{2}$ ImmunoX Initiative, and ${ }^{3}$ Biological Imaging Development CoLab, UCSF, San Francisco, California, USA.

\begin{abstract}
Intratumoral T cells that might otherwise control tumors are often identified in an "exhausted" state, defined by specific epigenetic modifications and upregulation of genes such as CD38, cytotoxic T-lymphocyte-associated protein 4 (CTLA4), and programmed cell death 1 (PD1). Although the term might imply inactivity, there has been little study of this state at the phenotypic level in tumors to understand the extent of their incapacitation. Starting with the observation that T cells move more quickly through mouse tumors the longer they reside there and progress toward exhaustion, we developed a nonstimulatory, live-biopsy method for the real-time study of T cell behavior within individual patient tumors. Using 2-photon microscopy, we studied native CD8+ $\mathrm{T}$ cell interaction with antigen-presenting cells (APCs) and cancer cells in different microniches of human tumors and found that $\mathrm{T}$ cell speed was variable by region and by patient and was inversely correlated with local tumor density. Across a range of tumor types, we found a strong relationship between CD8 ${ }^{+}$T cell motility and the exhausted T cell state that corresponded with our observations made in mouse models in which exhausted T cells moved faster. Our study demonstrates $\mathrm{T}$ cell dynamic states in individual human tumors and supports the existence of an active program in "exhausted" $T$ cells that extends beyond incapacitating them.
\end{abstract}

\section{Introduction}

Tumors contain inflammatory infiltrates that might detect and eliminate tumor cells, and yet immune tolerance occurs, and cancer evasion persists. T cells isolated from the tumor microenvironment (TME) often exhibit an "exhausted" phenotype characterized by a unique epigenetic landscape and increased inhibitory receptor expression that lead to reduced proliferative capability and tumor killing (1). Given that T cells are the targets for most current cancer immunotherapies, understanding how this tolerant state manifests in situ is important. Multiplexed flow cytometry and single-cell transcriptomics have been applied to isolated intratumoral $\mathrm{T}$ cells to investigate their function (2), however, the spatial information is lacking. In contrast, fixed imaging methods reveal important links between protein phenotypes and the spatial organization of immune infiltrates (3), but do not show the dynamic behaviors and the plasticity of cellular ensembles.

Intravital imaging by 2-photon laser microscopy - using near-infrared excitation to provide improved depth penetration with reduced phototoxicity over longer observation periods - is especially suited for in situ imaging of immune dynamics in intact tumors (4). We and others have established methods to study how immune cells behave in mouse tumors, often using genetically encoded fluorophores $(5,6)$. In one such setting, we found evi-

\section{Related Commentary: https://doi.org/10.1172/JCl152382}

Conflict of interest: The authors have declared that no conflict of interest exists. Copyright: @ 2021, American Society for Clinical Investigation.

Submitted: September 16, 2020; Accepted: July 20, 2021; Published: September 15, 2021 Reference information: / Clin Invest. 2021;131(18):e144353.

https://doi.org/10.1172/JCl144353. dence that infiltrated $\mathrm{T}$ cells increase their motility following an initial arrest phase (7). The behavior of T cells in human tumors which likely vary in myriad ways relative to mouse tumor models - remains largely unstudied.

In one pioneering study using slices of human biopsies, exogenous $\mathrm{T}$ cells labeled with fluorescent dyes that were non-native to tumors were imaged (8). There, these newly introduced cells migrated actively in loose collagen areas, but poorly in dense matrix areas. To our knowledge, however, no systemic approach has yet been developed to study how tumor-native $\mathrm{T}$ cells migrate or how that links to their immune function in human tumors. Therefore, we developed and validated a livebiopsy method for direct imaging of immune function in human tumor specimens. We standardized the approach and applied it to tumors of multiple cancer types to measure immune cell behavior as indicated by cell motility and interaction among antigen-presenting cells (APCs), T cells, and tumor cells. The results showed that there was heterogeneity within human cancers with respect to exhaustion and that the program of exhaustion defines a dynamic state resembling surveillance.

\section{Results and Discussion}

Development of live-biopsy imaging. To characterize the in situ phenotype of intratumoral high-affinity murine T cells, we generated a cell line from a well-described breast cancer mouse model (mammary tumor virus-polyoma middle T [MMTV-PyMT]) modified to express mCherry and OVA (PyMT-ChOVA) (5) and injected the cells into the fat pads of mice with the adoptive transfer of OT-I cells at 2 different time points during tumor progression (Figure 1A). T cells resident in tumors for 14 days were characterized by the upregulation of inhibitory molecules associated with 
A

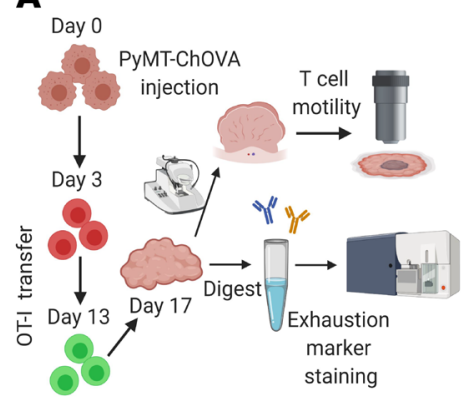

C

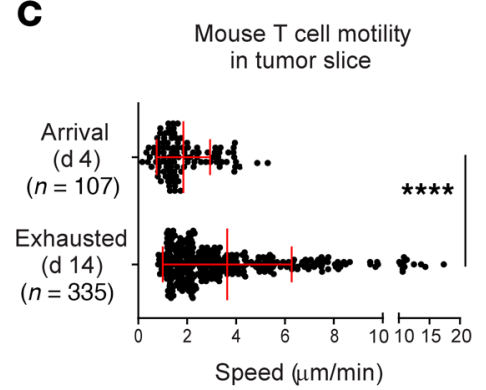

E

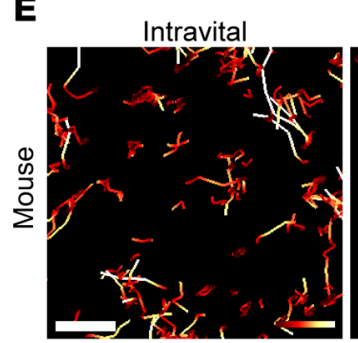

B
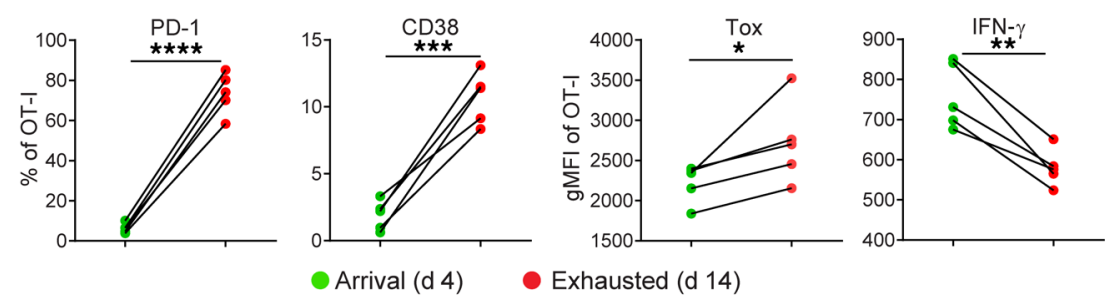

D

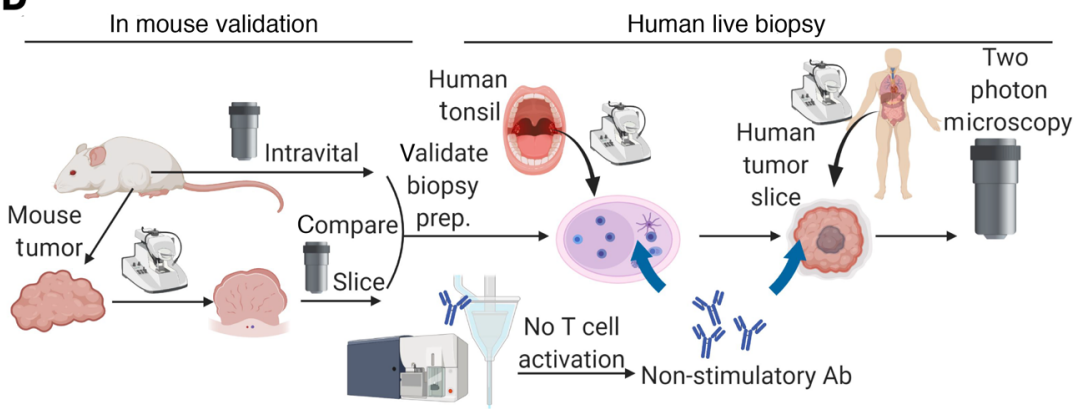


aid visualization and that do not trigger well-established signaling pathways downstream of their ligands (Figure 1D). For example, we selected a single clone, V $\beta 13.1$, that bound to a T cell receptor $\beta$ (TCR $\beta$ ) (Figure $1 \mathrm{~F}$ ) and an anti-CD $8 \alpha$ antibody (Supplemental Figure 1B) that did not induce a detectable calcium influx in Jurkat cells or in human PBMCs when cross-linked (Supplemental Figure 1C), unlike the majority of monoclonal antibodies against TCRassociated proteins (e.g., CD3, TCR, etc.), which did so. Where possible, we used quantum dot (Qdot) conjugates of these clones, given their exceptional brightness and photostability (11). As a functional test of this panel in situ, we examined human tonsil slices to visualize $\mathrm{CD}^{+} \mathrm{T}$ cells and $\mathrm{CD} 14^{+}$myeloid cells (Supplemental Figure 2A and Video 1) and found that the motility of human $\mathrm{T}$ cells fell within the range of 1 to $10 \mu \mathrm{m} / \mathrm{min}$ (Supplemental Figure 2B), consistent with mouse lymphocyte motility in lymph nodes (12). We observed diverse $\mathrm{CD}^{+} \mathrm{T}$ cell behaviors in a microregion of the tonsil, including some cells with a random walk and others that were arrested on myeloid cells (Supplemental Figure 2C), which might have been attributable to the presence of antigens in these inflamed tonsils.

Application of this method to tumors, including head and neck squamous cell (HNSC), colorectal (CRC), gynecological (GYN), kidney (KID), and hepatic (HEP) cancers (Supplemental Figure 3), showed well-maintained immune infiltrates, stroma, and tumor structure. An example of a HNSC tumor highlighted diverse levels of immune infiltration and $\mathrm{T}$ cell speeds among 5 different regions of interest (ROIs), chosen at random during acquisition (Supplemental Figure 4, A and B). Neither the density of $\mathrm{CD}^{+} \mathrm{T}$ cells or HLA-DR ${ }^{+}$myeloid cells, nor the contact time between them in these 5 ROIs was well correlated with $\mathrm{T}$ cell speed (Supplemental Figure 4, C-F).

$C D 8^{+} \mathrm{T}$ cell motility was inversely correlated with local tumor density. To extend our analysis to additional tumors and identify other tissue parameters that might affect motility, we imaged a colorectal tumor, in which $\mathrm{CD}^{+} \mathrm{T}$ cell behaviors in 3 ROIs with a varying abundance of $\mathrm{EpCAM}^{+}$tumor cells were quantified (Figure 2, A and B, and Supplemental Video 2). T cell tracks showed marked regional differences (Figure 2, C and D), but were not correlated with the density of either the $\mathrm{T}$ cells or the myeloid cells, or with the contact time between these 2 cell types (Supplemental Figure $5, \mathrm{~A}-\mathrm{C})$. However, tumor cell density was inversely correlated with $\mathrm{T}$ cell motility across the 3 ROIs (Figure 2, E and F). This relationship was repeated in another CRC (Supplemental Figure 5, D-G) and in the HNSC shown in Supplemental Figure 4 and Figure 2F.

Finally, we observed that, although most $\mathrm{CD}^{+} \mathrm{T}$ cells moved freely when they were not in contact with tumor cells, they occasionally alternatively engaged with myeloid cells and tumor cells to form stable conjugates for at least 90 minutes (Figure $2 \mathrm{G}$ and Supplemental Video 2 ). Thus, $\mathrm{CD}^{+} \mathrm{T}$ cell motility differed among tumor subregions, potentially because of heterogeneous local tumor density.

$C D 8^{+} T$ cell motility was correlated with immune states in human tumors. We next sought to broaden this approach toward defining the rate of $\mathrm{T}$ cell motility across tumors and across sites of origin. In total, 17 fresh tumors of 5 different cancer types were split for flow cytometry (Supplemental Figure 6A) and live-biopsy imaging (Figure 3A). Although $\mathrm{T}$ cell speed was not a binary variable, we classified $\mathrm{T}$ cells with substantial motility across the tumor (motile) versus those with very limited motility (Immotile) using a cutoff if the mean was less than $1 \mu \mathrm{m} / \mathrm{min}$ (Figure 3B), to investigate whether the immune component and cell state were linked to fast versus slow $\mathrm{T}$ cell speed. Although these groups had different ischemia durations (Supplemental Figure 6B), we found no statistical evidence that these were different between the motility groups. The average $\mathrm{CD} 8^{+} \mathrm{T}$ cell density, the percentage of $\mathrm{CD} 45^{+}$ live cells, the $\mathrm{CD}^{+}$to $\mathrm{CD} 8^{+} \mathrm{T}$ cell ratio, and the abundance of Tregs (Supplemental Figure 6, C-F) were also quantified for these samples, and all showed no relationship to motility.

We then turned to markers of exhaustion, guided by the mouse data (Figure 1), as a hypothesis. We examined the frequency of $\mathrm{CD}^{+} \mathrm{T}$ cells expressing the exhaustion markers CD38 and CTLA-4, and found that these markers were upregulated in the motile group compared with expression levels in the immotile group (Figure 3, C-F). PD-1 expression was also mildly upregulated in the motile group (Figure 3, G and $\mathrm{H}$ ).

We also sought to determine whether motility and exhaustion had other correlates and found that Ki67 expression in $\mathrm{CD}^{+} \mathrm{T}$ cells followed the same trend (Figure 3, I and J). Although Ki67 is often used as a marker for proliferative capability, it also marks a population of recently generated, terminal exhausted $\mathrm{T}$ cells that lose the ability to respond to additional stimulation (13). In the tumor biopsies we imaged, Ki67 ${ }^{\text {hi }} \mathrm{CD} 8^{+} \mathrm{T}$ cells had significantly higher expression of both PD-1 and CD38 than did $\mathrm{Ki}^{16} 7^{\mathrm{lo}} \mathrm{CD} 8^{+} \mathrm{T}$ cells from the same tumors (Supplemental Figure 6G).

To further examine the link between $\mathrm{T}$ cell exhaustion and motility, we performed RNA-Seq of T cells sorted from a cohort of patients with CRC, one of the most abundant cancer types analyzed by live biopsy, and grouped the samples on the basis of CD38 upregulation as a representative marker for exhausted T cells. ENTPD1, LAG3, HAVCR2, and TOX2 were upregulated, supporting the idea that these cells were preferentially exhausted, whereas $T N F$ was downregulated, indicating a reduced effector phenotype (Figure 4A). Referencing gene ontology of the differentially expressed genes also showed that protein localization to microtubule and regulation of cell migration-related pathway genes were both enriched in the CD38 ${ }^{\text {hi }}$ group (Figure $4 \mathrm{~B})$. These included genes associated with cytoskeletal rearrangement and cell polarization (PDLIM4,AFAP1L2, and PLK4), microtubule motor protein-encoding genes, (KIF20A, MYO7A, and $M Y O 7 B$ ), and the Rho-GTPase-associated protein caveolin 1 (CAV1) (Figure 4A). We then plotted the fold-change for these promotility genes revealed in our data by comparing "exhausted" versus "nonexhausted" cells on the basis of the RNA-Seq data from 4 recent publications on exhaustion, and found that all of these genes were upregulated in exhausted T cells (Figure 4C) in human lung cancer and melanoma (14-17). Together, these observations show that "exhausted" cells, though defective for sensitivity and the ability to mount cytokine responses, nevertheless show upregulation of genes associated with tissue surveillance.

For a direct comparison of exhaustion markers with cell motility on a cell-by-cell basis, we monitored T cell motility and expression of exhaustion markers simultaneously in PyMT tumor slices (Figure 4D). We found that long-resident $\mathrm{T}$ cells expressed 
A

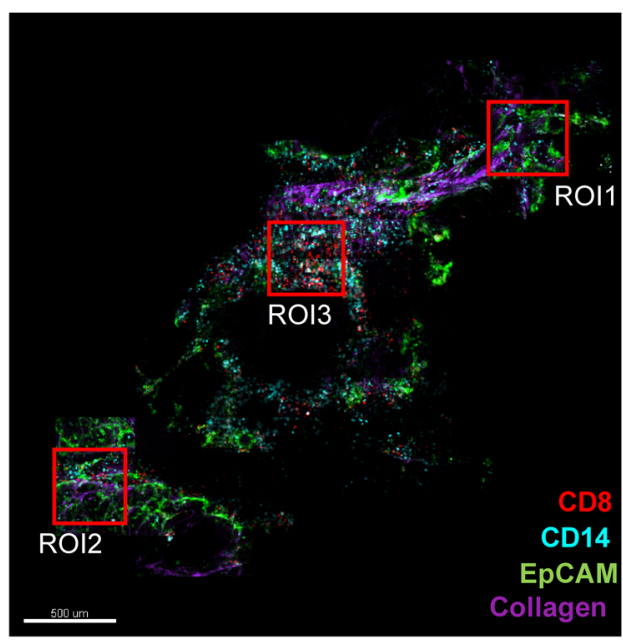

B

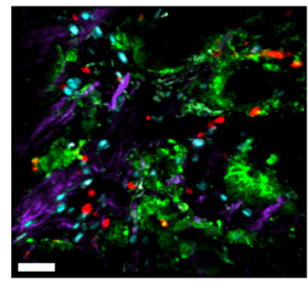

C

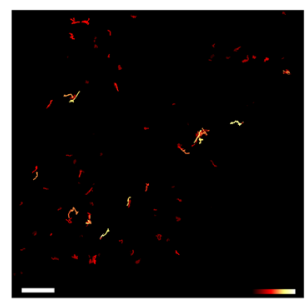

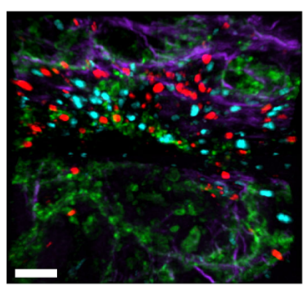

ROI2

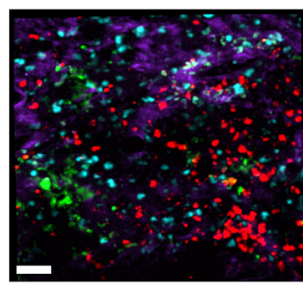

$\mathrm{ROI} 3$
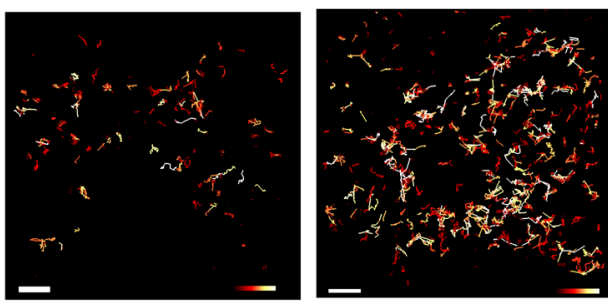

D

E

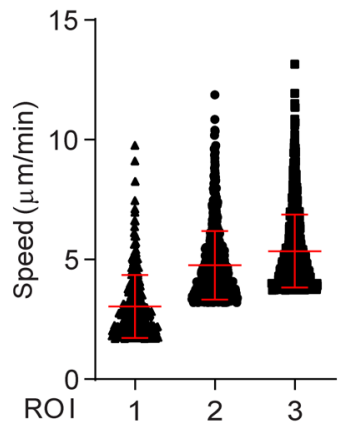

$\mathrm{EpCAM}^{+}$

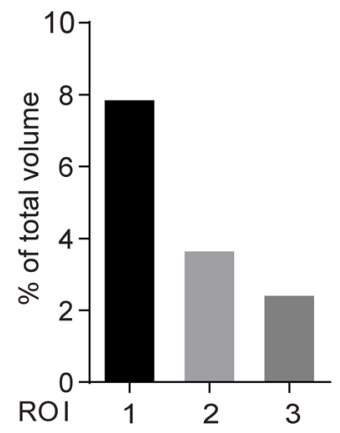

$\mathbf{F}$

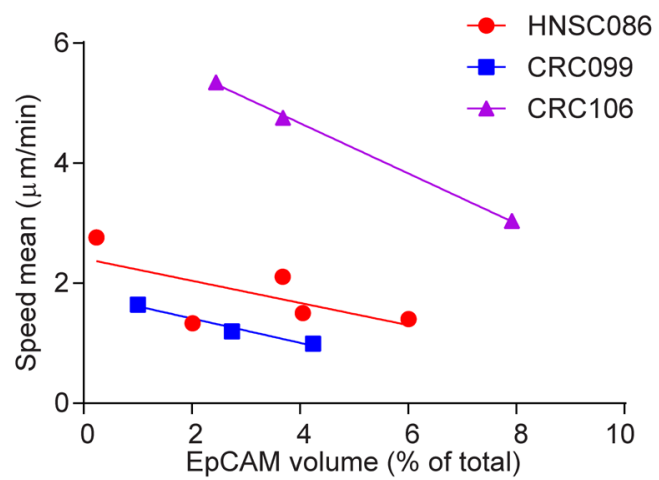

G
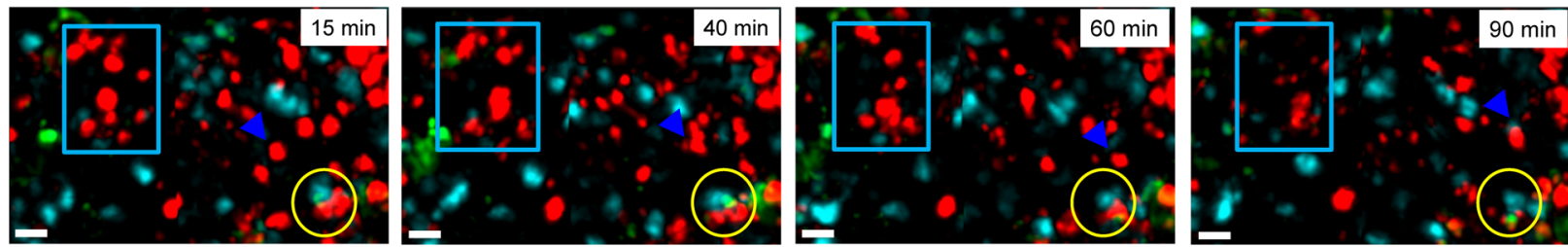

CD8 CD14 EpCAN

Figure 2. Inverse correlation of CD8+ $\mathbf{T}$ cell motility with tumor density in cancer biopsies. (A) A scan of a colorectal cancer slice. Scale bar: $500 \mu \mathrm{m}$. (B) Three ROls from panel A. Scale bars: $50 \mu \mathrm{m}$. (C) Color-coded track displacement of CD8 ${ }^{+}$T cells. Length range: $0.5 \mu \mathrm{m}$ (red) to $25 \mu \mathrm{m}$ (white). Scale bars: $50 \mu \mathrm{m}$. (D) The speed of the top $10 \%$ fastest CD8 $8^{+}$T cells was plotted. Data indicate the mean \pm SD. (E) Percentage of the EpCAM ${ }^{+}$volume in the total volume. (F) The mean speed of CD8 ${ }^{+} T$ cells from 3 tumor samples plotted, respectively, against the tumor cell density of the corresponding ROls from the biopsy. (G) Time-lapse images of a video recording of ROI2 showing CD8 ${ }^{+} \mathrm{T}$ cell movement (blue rectangles) and a T cell that moved to a CD14+ cell (blue arrowheads). T-APC-tumor cell conjugates remained through 90 minutes (yellow circles). See also Supplemental Video 2.

higher PD-1 levels than did recently arrived T cells (Figure 4E) and moved faster with a longer track (Figure $4 \mathrm{~F}$ ). When quantifying these parameters in individual cells from tumors of the same volume, day- $14 \mathrm{~T}$ cells exhibited higher PD-1 expression and speed, whereas day- $4 \mathrm{~T}$ cells expressed lower levels of PD-1 and were slower, with a positive overall correlation between $\mathrm{T}$ cell motility and exhaustion (Figure 4G).

In summary, we developed a 2-photon microscopy-based method for imaging tumor slices from fresh biopsies obtained from cancer patients that maintained immune dynamics and spatio- temporal information. Our study, focused on in situ endogenous $\mathrm{T}$ cell behavior in human cancer, provides a view of regional and patient-specific variations in cellular dynamics and furthers our understanding of a critical T cell state, namely that of "exhaustion."

With regard to methods development, the biology observed using these imaging conditions and nonstimulatory antibodies strongly resembles that observed in mouse lymph nodes and in situ $(4,5,12)$, suggesting that we have broadly captured the durable biology of these tissues. Although the full-size immunoglobulins used in our methods were nondisturbing, using a monovalent Fab 
A

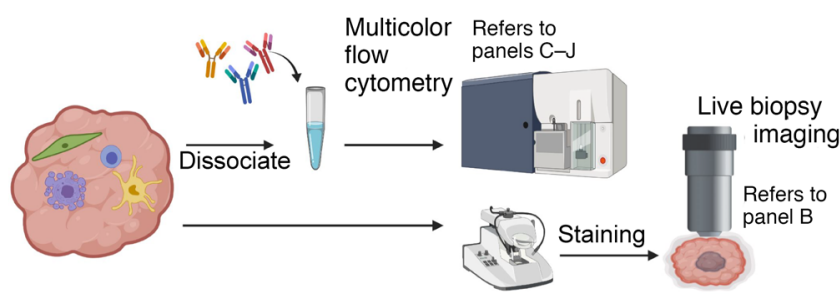

C

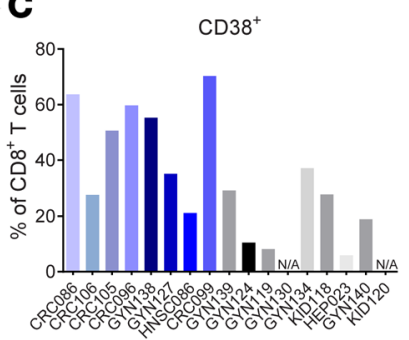

D

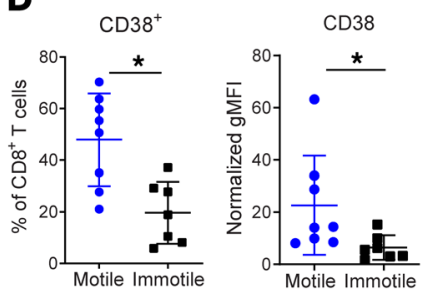

G
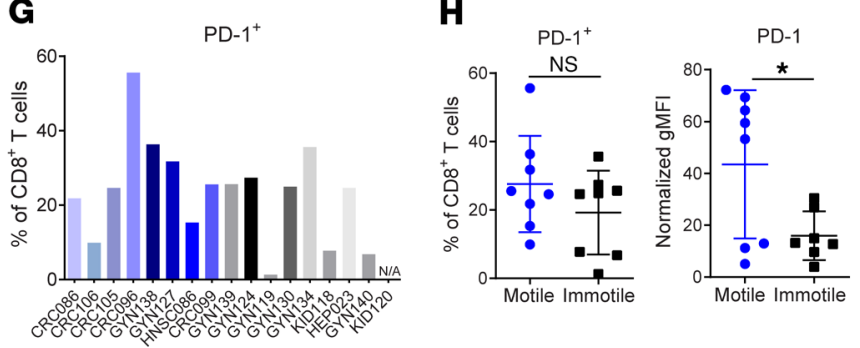

B

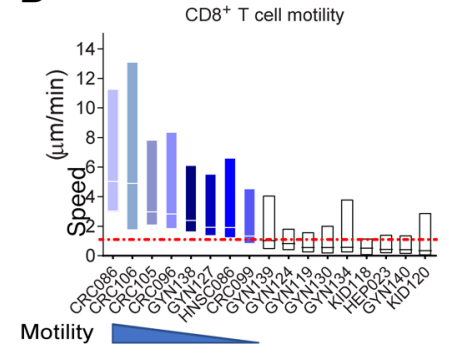

E

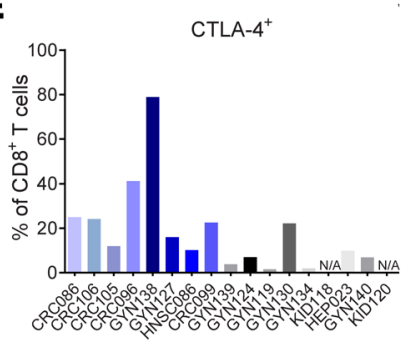

$\mathbf{F}$

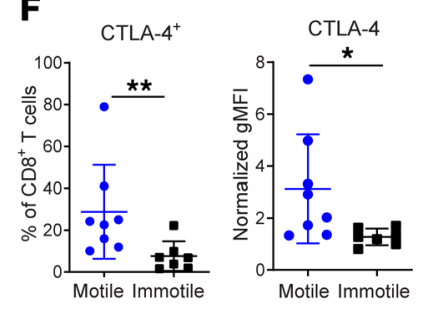

I

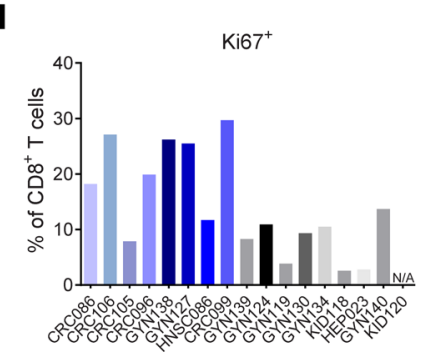

J

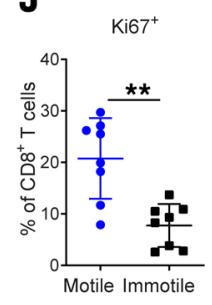

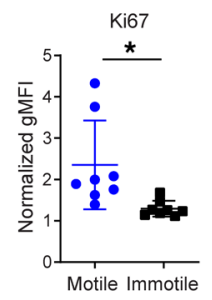

Figure 3. Linkage of T cell motility and exhaustion phenotypes in human tumors. (A) Diagram of a fresh tumor split for live-biopsy imaging and flow cytometry. (B) CD8 $\mathrm{T}$ cell speed across multiple tumor biopsies. Blue bars represent specimens with motile T cells; black/gray bars represent immotile T cells (speed mean $<1 \mu \mathrm{m} / \mathrm{min}$, indicated by the red dashed line). Each bar (minimum to maximum with the line at the mean) represents the CD $8^{+} \mathrm{T}$ cell motility data pooled from 3 or more ROls in 1 biopsy. (C-J) Flow cytometry of cells from the same tumor biopsies as in B. Percentage data are plotted as bar graphs, and dot plots show normalized geometric MFIs of motile versus immotile groups for comparison. Each dot represented 1 biopsy. ${ }^{*} P<0.05$ and ${ }^{* *} P$ $<0.01$, by Student's $t$ test. $n=17$.

or nanobodies to eliminate Fc region-medicated effects would be ideal. Despite the fact that we optimized our slice imaging method, it is possible that immune behavior was not perfectly recapitulated, because vascular circulation and a chemokine milieu in addition to enervation and lymphatic flow were lacking (18).

Regardless of the different histological tumor types and their genetic variations, the combination of immune profiling by flow cytometry/RNA-Seq and live-biopsy imaging enabled us to group tumors according to $\mathrm{T}$ cell motility and link these data to the aggregated state of the $\mathrm{T}$ cell population. In our study, the aggregated abundance of human exhausted T cells marked with Ki67 ${ }^{\text {hi } C T L A-~}$ $4^{\text {hi }} \mathrm{PD}-1^{\text {hi }} \mathrm{CD} 38^{\text {hi }}$ correlated well with the observation of fast motility within the same tumor sample. Although multiple factors could contribute to the progression of $\mathrm{T}$ cell motility with tumor residence and during transitions toward exhaustion, including varied $\mathrm{T}$ cell priming in the lymph nodes and desensitization to the existence of tumor antigens (19), our data suggested that T cells exhibited the exhausted phenotype gradually with a wide spectrum of exhaustion markers expressed at different levels. Correspondingly, although T cell speed within a given human sample varied, the overall motility was positively correlated with an exhausted phenotype. Given the observation of an absolute shift in motility pattern during the establishment of exhaustion in mouse models (Figures 1 and 4), we believe that this relationship is probably at least partially cell intrinsic. This is further suggested by the upregulation of motility genes in $T$ cells obtained from patients with an abundance of exhausted $\mathrm{T}$ cells. Further analysis of single-cell sequencing data on exhausted $\mathrm{T}$ cells from patient samples may reinforce this finding, as could in vitro experiments with $\mathrm{T}$ cells taken from patients. However, we consider it likely that this faster motility rate is also a consequence of the combination of cell-intrinsic and -extrinsic features and may even represent the coevolution of microenvironmental features along with the induction of exhaustion. Our live-biopsy method makes it possible to test whether TCR-HLA interaction blockade could lead to an increase in motility or, in contrast, whether exogenous peptide or anti-PD-1 blockade could arrest exhausted T cells in tumors. It will be intriguing to explore how other microregional factors, including cell types, soluble factors, local oxygen levels, protease, and metabolites, regulate immune cell behavior and $\mathrm{T}$ cell function, e.g., exhaustion. To understand all these factors together, many more studies will be required, notably those involving spatial transcriptomics and/or proteomics. 
A

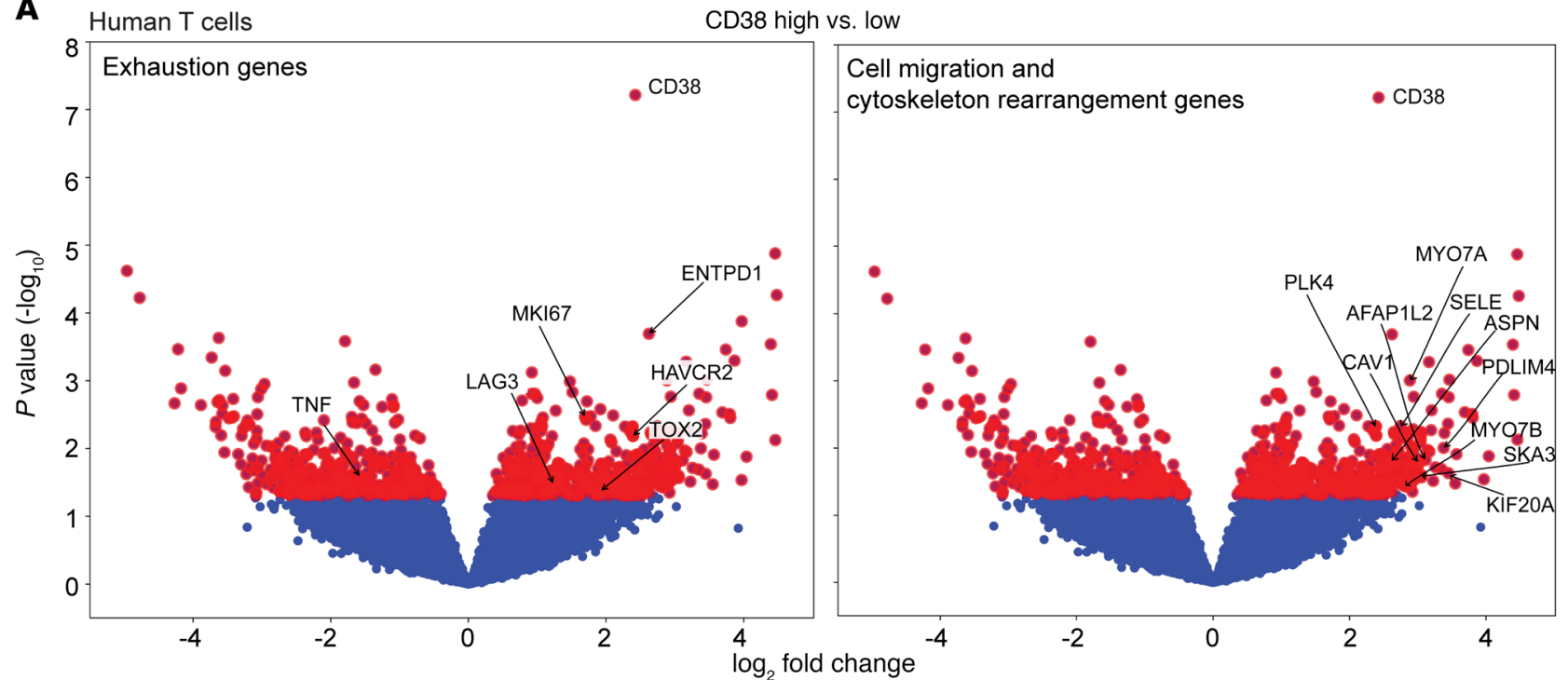

B

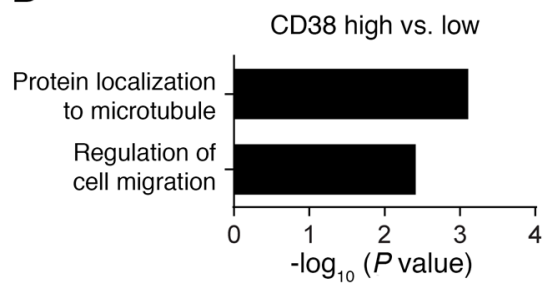

E

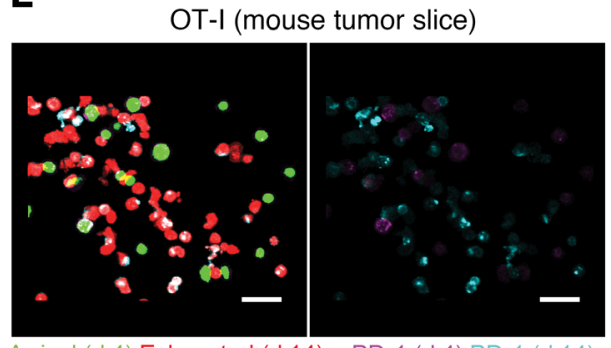

C

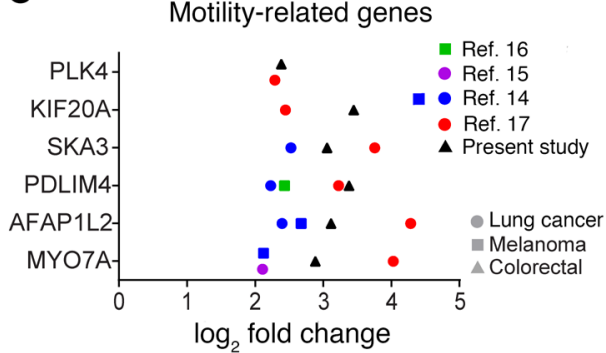

$F$

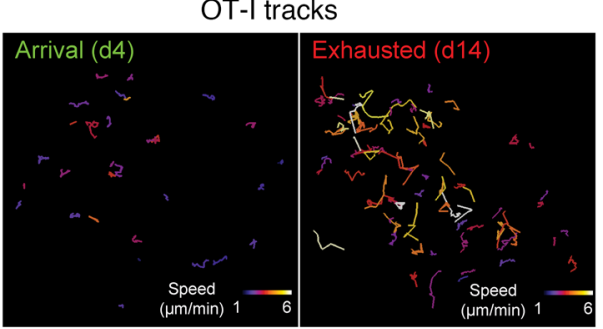

D Day 0

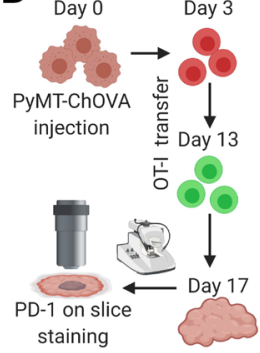

G

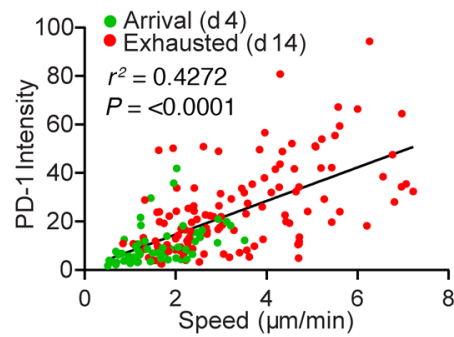

Figure 4. Exhausted T cells exhibit motile features. (A) Volcano plots of differentially expressed genes between CD38 ${ }^{\text {hi }}$ (top 33 rd percentile of all samples) and CD38 ${ }^{\circ}$ (bottom 33rd percentile) CRC samples. Red dots are above the cutoff for $P=0.05$. (B) Gene ontology analysis of the upregulated genes in the CD38 ${ }^{\text {hi }}$ versus $C D 38^{\text {lo }}$ samples. (C) Fold-change of promotility genes in exhausted versus nonexhausted T cells from RNA-Seq of melanoma or lung cancer samples as reported in refs. 14-17. (D) Schematic diagram of the experimental design for E-C. (E) Representative images of PD-1 staining for RFP (d14) and GFP in OT-I cells (d4) that resided in the same field. Scale bars: $30 \mu \mathrm{m}$. (F) Speed-based, color-coded track displacement of GFP and RFP OT-I cells. (G) PD-1 intensity on OT-I cells was plotted against the mean speed of each cell. Data were pooled from 3 different ROls and are representative of 2 independent experiments. $P$ and $r^{2}$ values were obtained by linear regression analysis.

Given that the composition and type of immune cells in the TME affect clinical outcomes (20), the live-biopsy method developed here will provide a platform to test drugs, e.g., immune checkpoint inhibitors in tumors demonstrating these distinct $\mathrm{T}$ cell phenotypes. Following the disseminated use and elaboration of these methods, live-biopsy studies may serve as "mini-patients" to predict the efficacy of the same therapy in patients.

\section{Methods}

Detailed experimental methods and statistical analyses are included in the Supplemental Methods.
The RNA-Seq data reported here are deposited in the NCBI's Gene Expression Omnibus (GEO) database (GEO GSE179975).

Study approval. Patients in this study provided written informed consent for tissue collection under a UCSF IRB-approved protocol (no. 13-12246). All mice were housed and bred at UCSF in accordance with the regulatory standards of the NIH and the American Association of Laboratory Animal Care, and their treatment was consistent with UCSF IACUC standards (no. AN170208-01B).

\section{Author contributions}

RY performed the majority of the experiments. JA, AF, and AWE 
assisted with imaging analysis. AJC and GCR participated in the preparation and analysis of the flow cytometric experiments involving human samples. BS processed and analyzed RNA-Seq data. RY and MFK designed the experiments and wrote and revised the manuscript.

\section{Acknowledgments}

We thank the Biological Imaging Development Colab for microscopy data collection and instrumentation; the Parnassus Flow
Cytometry Core for instrumentation (supported by grant no. P30DK063720); and the Institute for Human Genomics for sequencing and bioinformatics support at UCSF. This work was supported in part by the National Cancer Institute (NCI), NIH (R21CA196468, to MFK).

Address correspondence to: Matthew F. Krummel, 513 Parnassus Avenue, HSW 512, San Francisco, California 94143-0511, USA. Phone: 415.514.3130; Email: matthew.krummel@ucsf.edu.
1. Blank CU, et al. Defining 'T cell exhaustion'. Nat Rev Immunol. 2019;19(11):665-674.

2. Huang AC, et al. T-cell invigoration to tumour burden ratio associated with anti-PD-1 response. Nature. 2017;545(7652):60-65.

3. Keren L, et al. A structured tumor-immune microenvironment in triple negative breast cancer revealed by multiplexed ion beam imaging. Cell. 2018;174(6):1373-1387.

4. Bullen A, et al. Two-photon imaging of the immune system: a custom technology platform for high-speed, multicolor tissue imaging of immune responses. Curr Top Microbiol Immunol. 2009;334:1-29.

5. Engelhardt JJ, et al. Marginating dendritic cells of the tumor microenvironment cross-present tumor antigens and stably engage tumor-specific T cells. Cancer Cell. 2012;21(3):402-417.

6. Boissonnas A, et al. In vivo imaging of cytotoxic $\mathrm{T}$ cell infiltration and elimination of a solid tumor. JExp Med. 2007;204(2):345-356.

7. Boldajipour B, et al. Tumor-infiltrating lymphocytes are dynamically desensitized to antigen but are maintained by homeostatic cytokine. JCI Insight. 2016;1(20):e89289.

8. Salmon $\mathrm{H}$, et al. Matrix architecture defines the preferential localization and migration of $\mathrm{T}$ cells into the stroma of human lung tumors. J Clin Invest. 2012;122(3):899-910.

9. Philip M, et al. Chromatin states define tumour-specific T cell dysfunction and reprogramming. Nature. 2017;545(7655):452-456.

10. Khan O, et al. TOX transcriptionally and epigenetically programs $\mathrm{CD} 8^{+} \mathrm{T}$ cell exhaustion. Nature. 2019;571(7764):211-218.

11. Larson DR, et al. Water-soluble quantum dots for multiphoton fluorescence imaging in vivo. Science. 2003;300(5624):1434-1436.

12. Hugues S, et al. Distinct T cell dynamics in lymph nodes during the induction of tolerance and immunity. Nat Immunol. 2004;5(12):1235-1242.

13. Paley MA, et al. Progenitor and terminal subsets of $\mathrm{CD}^{+} \mathrm{T}$ cells cooperate to contain chronic vira infection. Science. 2012;338(6111):1220-1225.

14. Li H, et al. Dysfunctional CD8 T cells form a proliferative, dynamically regulated com- partment within human melanoma. Cell. 2019;176(4):775-789.

15. Guo X, et al. Global characterization of T cells in non-small-cell lung cancer by single-cell sequencing. Nat Med. 2018;24(7):978-985.

16. Miller BC, et al. Subsets of exhausted CD8(+) $T$ cells differentially mediate tumor control and respond to checkpoint blockade. Nat Immunol. 2019;20(3):326-336.

17. Thommen DS, et al. A transcriptionally and functionally distinct PD- $1^{+} \mathrm{CD}^{+} \mathrm{T}$ cell pool with predictive potential in non-small-cell lung cancer treated with PD-1 blockade. Nat Med. 2018;24(7):994-1004.

18. Benechet AP, et al. Visualizing T cell migration in situ. Front Immunol. 2014;5:363.

19. Honda T, et al. Tuning of antigen sensitivity by $\mathrm{T}$ cell receptor-dependent negative feedback controls $\mathrm{T}$ cell effector function in inflamed tissues. Immunity. 2014;40(2):235-247.

20. Fridman WH, et al. The immune contexture in human tumours: impact on clinical outcome. Nat Rev Cancer. 2012;12(4):298-306. 